\title{
PULMONARY VASOREACTIVITY AND PHENOTYPES IN PULMONARY ARTERIAL HYPERTENSION ASSOCIATED TO CONNECTIVE TISSUE DISEASES
}

\author{
José luis Hernández-Oropeza ${ }^{1 *}$, Tatiana Sofía Rodríguez-Reyna², Diego luis Carrillo-Pérez ${ }^{3}$, \\ José de Jesús Rodríguez-Andoney ${ }^{1}$, René Narváez-David ${ }^{4}$, Yesenia Salado-Morales ${ }^{1}$, \\ Eduardo Rivero-Sigarroa ${ }^{5}$, Guillermo Domínguez-CheritT ${ }^{5}$ and Tomás Pulido-Zamudio ${ }^{6}$ \\ ${ }^{1}$ Department of Cardiopulmonary Disease, ${ }^{2}$ Department of Immunology and Rheumatology, ${ }^{3}$ Department of Internal \\ Medicine, ${ }^{4}$ Department of Cardiology, and ${ }^{5}$ Department of Critical Care Medicine, Instituto Nacional de Ciencias \\ Médicas y Nutrición Salvador Zubirán; and ${ }^{6}$ Department of Cardiopulmonary Disease, Instituto Nacional de Cardiología \\ Ignacio Chávez, Mexico City, Mexico
}

\begin{abstract}
Background: Pulmonary arterial hypertension (PAH) is a fatal complication in patients with connective tissue disease (CTD). Objective: The objective of the study was to study the prognostic value of the acute pulmonary vasoreactivity test with inhaled iloprost and its association with clinical deterioration in a tertiary care academic medical center. Methods: We conducted a prospective study of patients with CTD and the diagnosis of PAH established by right heart catheterization. Patients were classified into classic responders, partial responders, and non-responders. The association of the pulmonary response and clinical deterioration was analyzed. Results: We enrolled 25 patients (mean age of $47 \pm 13.4$ years); $88 \%$ were female. The most frequent rheumatologic diagnosis was systemic lupus erythematosus, in 16 (64\%) patients. Seventy-two percent of patients were classified as non-responders, and $28 \%$ were partial responders. Patients with a partial response had lower right atrial pressure values $(5.1 \pm 3.1$ vs. $8.5 \pm 3.2, p=0.01)$ and greater systolic pulmonary arterial pressure $(87.6 \pm 8.1$ vs. $72.4 \pm 16.2$, $p=0.02$ ), compared with non-responders. Non-responders had a tendency for a shorter time to clinical deterioration than partial responders ( 17.8 vs. 41.1 months, $p=0.052$ ). Conclusions: Patients with a partial response to the acute pulmonary vasodilator test with inhaled iloprost had a longer clinical deterioration-free period than non-responders. (REV INVES CLIN. 2018;70:82-7)
\end{abstract}

Key words: Pulmonary hypertension. Right heart catheterization. Connective tissue disease. Prognosis.

Corresponding author:

*José Luis Hernández-Oropeza

Department of Cardiopulmonary Disease

Instituto Nacional de Ciencias Médicas

y Nutrición Salvador Zubirán

Vasco de Quiroga, 15

Col. Sección XVI, Del. Tlalpan

C.P. 14080, Mexico City, Mexico

Received for publication: 08-11-2017

E-mail: elinternista@hotmail.com

Accepted for publication: 13-02-2018

doi: $10.24875 / R I C .18002437$ 


\section{INTRODUCTION}

Pulmonary arterial hypertension (PAH) is characterized by the progressive increase in pulmonary vascular resistance, leading to right ventricular dysfunction. Right heart catheterization ( $\mathrm{RHC}$ ) establishes the diagnosis of PAH and guides the most appropriate medical therapy according to the patient's response to the acute pulmonary vasoreactivity test (APVT) with short-acting vasodilators (nitric oxide, epoprostenol, adenosine, and iloprost $)^{1,2}$.

$\mathrm{PAH}$ is present in $13-25 \%$ of patients with connective tissue disease (CTD), and it is a major cause of death in this sub-group ${ }^{3}$. The use of calcium channel blockers is precluded in this population since these patients are considered non-responders in the long-term ${ }^{4-6}$.

Recent studies have evaluated the predictive usefulness of the APVT in individuals with PAH in association with CTD (PAH-CTD), in partial responders with contoversial prognostic results 7,8 .

The aim of this study was to determine the prognostic value of the APVT with inhaled iloprost in patients with PAH-CTD, according to the response in the test. We have hypothesized that patients with some type of pulmonary vascular response would present fewer events compatible with clinical deterioration.

\section{PATIENTS AND METHODS}

\section{Study population}

We conducted a prospective study of patients with CTD in whom, as part of their standard medical care, RHC was required, and an APVT was not contraindicated. The study was performed in a tertiary care academic medical center in Mexico City, which is a referral center for CTD, between January 2010 and December 2015.

The following variables were analyzed: clinical characteristics, lung function tests, biomarkers of right ventricular dysfunction, stress test, and hemodynamic parameters detected by echocardiography and hemodynamic testing.

\section{Definitions}

Patients were considered to have PAH if their mean pulmonary arterial pressure (mPAP) was $\geq 25 \mathrm{mmHg}$, the pulmonary capillary pressure was $<15 \mathrm{mmHg}$, and pulmonary vascular resistance was $>3$ Wood units, according to RHC results ${ }^{1,2}$. Patients with CTD were those cases with a clinical and immunological diagnosis of systemic lupus erythematosus (SLE), mixed CTD (MCTD), or systemic sclerosis (SS), all confirmed by the rheumatology department. Inhaled iloprost ( $5 \mathrm{mcg}$ ) was used in all cases to conduct the APVT during $\mathrm{RHC}^{1,9}$. A classic positive response was defined as the decrease of at least $10 \mathrm{mmHg}$ in the mPAP to an absolute value of $<40 \mathrm{mmHg}$ with an increased or unchanged cardiac output. A partial positive response referred to a decrease of at least $10 \mathrm{mmHg}$ to an absolute mPAP $>40 \mathrm{mmHg}$ with an increased or unchanged cardiac output Non-responders were those patients who fulfilled none of these criteria ${ }^{7}$.

In accordance with current definitions ${ }^{10}$, att patients were considered prevalent cases since both the CTD and the PAH had been diagnosed for over 6 months.

Clinical deterioration was defined as worsening of $\mathrm{PAH}$ (worsening symptoms and signs of right-sided heart failure), decrease in functional class, and decrease of at least $15 \%$ in the distance covered in the 6-minute walk test (6MWT) (confirmed by a second test 2 weeks later), or death.

\section{Statistical analysis}

Descriptive statistics were used according to the variables' characteristics. Frequencies and percentages were used for categorical variables, and continuous variables were reported as means and standard deviations, or medians, and interquartile ranges. The normality of the variables' distribution was evaluated with the Kolmogorov-Smirnov and the Shapiro-Wilk tests. comparison of continous variables were performed with Student's t-test and Wilcoxon's ranked sum test and the comparison of categorical variables were performed with Chi-square and Fisher's exact tests. Survival was analyzed with the Log-rank test. $p<0.05$ was considered statistically significant. Statistical analysis was performed with the STATA statistical package, version 11 . 
Table 1. Demographic characteristics, stress test, and lung function test results

\begin{tabular}{|c|c|c|c|}
\hline Variable & $\begin{array}{l}\text { Non-responder } \\
\quad(n=18)\end{array}$ & $\begin{array}{l}\text { Partial responder } \\
\qquad(\mathrm{n}=7)\end{array}$ & $\mathrm{p}$ \\
\hline Female, n (\%) & $16(89)$ & $6(86)$ & 0.82 \\
\hline BMI, $\left(\mathrm{kg} / \mathrm{m}^{2}\right)^{\mathrm{a}}$ & $23.5 \pm 4.2$ & $25.4 \pm 4.4$ & 0.32 \\
\hline SLE, n (\%) & $12(48)$ & $4(16)$ & 0.65 \\
\hline SS, n (\%) & $5(20)$ & $1(4)$ & 0.18 \\
\hline MCTD, n (\%) & $1(4)$ & $2(8)$ & 0.65 \\
\hline FC, I/II, n (\%) & $14(78)$ & $7(100)$ & 0.29 \\
\hline Distance $6 \mathrm{MWT}, \mathrm{m}^{\mathrm{a}}$ & $359 \pm 75.0$ & $386 \pm 55.4$ & 0.39 \\
\hline BNP, pg/dlb & $154(80-600)$ & $142(60-387)$ & $0.5 \overline{6}$ \\
\hline FVC, $(\%)^{\mathrm{a}}$ & $83.9 \pm 8.6$ & $80.5 \pm 6.4$ & 0.36 \\
\hline FEV1/FVC (\%) & & & 4 \\
\hline Ratio & $84.5 \pm 4.7$ & $84.7 \pm 4.9$ & 0.99 \\
\hline $\mathrm{PaO}_{2}, \mathrm{mmHg}^{\mathrm{a}}$ & $67.4 \pm 6.7$ & $67.42 \pm 9.6$ & 0.99 \\
\hline
\end{tabular}

BMI: body mass index, SLE: systemic lupus erythematosus, SS: systemic sclerosis, MCTD: mixed connective tissue disease, FC: functional class, 6MWT: 6-minute walk test, BNP: brain natriuretic peptide, FVC: forced vital capacity, FEV1: forced expiratory volume in 1 second; $\mathrm{PaO}_{2}$ : arterial oxygen pressure

aExpressed as a mean \pm standard deviation

bexpressed as a median and interquartile range

\section{RESULTS}

We included 25 patients throughout the study period, of whom 22 (88\%) were female. $16(64 \%)$ patients had a diagnosis of SLE, 6 (24\%) had SS, and 3 (12\%) had MCTD. In the SLE group, 13 (81\%) patients were on prednisone therapy, 3 (12\%) were treated with mycophenolate mofetil, and their average disease activity index according to the SLE disease activity index was $6.8 \pm 3.9$.

Demographic, clinical and laboratory characteristics are shown in table 1 . None of the patients fulfilled classic responder criteria. 18 patients (72\%) were considered non-responders and $7(28 \%)$ had a partial positive response. The mean age of the partial positive response group was $40 \pm 12.5$ years versus $49.3 \pm$ 13.2 years of the non-responder group.

Twenty-one (84\%) patients were WHO functional Class I and II, and 4 (16\%) were class III. Simple spirometry revealed a mildly restrictive pattern for both groups, and no interstitial involvement was observed by high-resolution tomography in any patient.

We detected the presence of anti-U1 ribonucleoprotein (RNP) antibodies in 4 patients ( $16 \%$ ), and 7 ( $28 \%$ ) patients were receiving low-dose calcium channel blockers (CCBs) for the management of Raynaud's phenomenon (nifedipine, $10 \mathrm{mg}$ twice a day). By Cox analysis, we found an association of 3 protective factors against clinical deterioration: a partial pulmonary vascular response, with an hazard ratio (HR) of 0.36 [95\% confidence interval $(\mathrm{Cl}) 0.021-1.302$ ]; presence of anti-U1-RNP antibodies, HR $0.50(95 \% \mathrm{Cl}$ $0.032-1.403)$; and the use of $C C B, H R$ of 0.34 (95\% $\mathrm{Cl} 0.042-2.780)$.

Hemodynamic characteristics of patients by echocardiography and cardiac catheterization are shown in table 2. In partial responders, the percentage of change in peripheral vascular resistance and mPAP was $21 \%$, with an unchanged cardiac output. Patients with a partial response had lower right atrial pressure values ( $5.1 \pm 3.1$ vs. $8.5 \pm 3.2, p=0.01)$ and greater systolic pulmonary arterial pressure $(87.6 \pm 8.1 \mathrm{vs}$. $72.4 \pm 16.2, p=0.02$ ), compared with non-responders. There were no statistically significant differences in other hemodynamic parameters.

All patients were administered supplemental oxygen as initial therapy (standard treatment in our center) and specific treatment with sildenafil monotherapy (25 mg PO every $8 \mathrm{~h}$ ) after PAH confirmation by RHC.

The median time period to clinical deterioration of the 3 CTD sub-groups was 17.8 months (range, 1.3-58.5 months) in the non-responder group versus 41.1 months (range, 12.5-59.5 months) in the partial responder group $(p=0.052$ ). Figure 1 shows the survival 
Table 2. Hemodynamic characteristics by echocardiography and cardiac catheterization

\begin{tabular}{|c|c|c|c|}
\hline Variable & $\begin{array}{l}\text { Non-responder } \\
\quad(n=18)\end{array}$ & $\begin{array}{l}\text { Partial responder } \\
\qquad(\mathrm{n}=7)\end{array}$ & $\mathbf{p}$ \\
\hline Echocardiography & & & 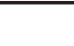 \\
\hline Systolic PAP, $(\mathrm{mmHg})^{\mathrm{a}}$ & $71.3 \pm 18.4$ & $70.1 \pm 21.6$ & 0.88 \\
\hline $\operatorname{MTRV},(\mathrm{m} / \mathrm{s})^{\mathrm{a}}$ & $3.6 \pm 0.7$ & $3.8 \pm 0.6$ & 0.49 \\
\hline TAPSE, $(\mathrm{mm})^{\mathrm{a}}$ & $18.8 \pm 2.9$ & $16.9 \pm 7.1$ & 0.32 \\
\hline RVSF, $(\%)^{\mathrm{a}}$ & $36.6 \pm 10.3$ & $35.7 \pm 10$ & 0.85 \\
\hline S wave, $(\%)^{\mathrm{a}}$ & $10.8 \pm 3.0$ & $10.5 \pm 3.1$ & 0.81 \\
\hline $\operatorname{LVEF},(\%)^{\mathrm{a}}$ & $64.4 \pm 9.7$ & $61.7 \pm 10.8$ & 0.54 \\
\hline Pericardial effusion, $\mathrm{n}(\%)$ & $6(24)$ & $2(8)$ & 0.89 \\
\hline \multicolumn{4}{|l|}{ Hemodynamics } \\
\hline Systolic PAP (mmHg) & $72.4 \pm 16.2$ & $87.6 \pm 8.1$ & 0.02 \\
\hline Diastolic PAP $(\mathrm{mmHg})^{\mathrm{a}}$ & $29.8 \pm 10.7$ & $36.4 \pm 4.4$ & 0.12 \\
\hline mPAP, $(\mathrm{mmHg})^{\mathrm{a}}$ & $46.6 \pm 12.1$ & $53.9 \pm 11.7$ & $0.1 \overline{2}$ \\
\hline $\mathrm{RAP},(\mathrm{mmHg})^{\mathrm{a}}$ & $8.5 \pm 3.2$ & $5.1 \pm 3.1$ & 0.01 \\
\hline $\mathrm{PCP},(\mathrm{mmHg})^{\mathrm{a}}$ & $9.2 \pm 3.1$ & $8.9 \pm 4.5$ & 0.55 \\
\hline PVR, (Wood units) ${ }^{\mathrm{a}}$ & $9.2 \pm 5$ & $13.2 \pm 6.6$ & 0.11 \\
\hline $\mathrm{Cl},\left(\mathrm{I} / \mathrm{min} / \mathrm{m}^{2}\right)^{\mathrm{a}}$ & $3.0 \pm 0.9$ & $2.8 \pm 0.8$ & 0.33 \\
\hline
\end{tabular}

PAP: pulmonary arterial pressure, MTRV: maximal tricuspid regurgitation velocity, TAPSE: tricuspid annular pulmonary systolic excursion, RVSF: right ventricular shortening fraction, LVEF: left ventricular ejection fraction, mPAP: mean pulmonary arterial pressure, RAP: right atrial pressure, PCP: pulmonary capillary pressure, PVR: pulmonary vascular resistance, $\mathrm{Cl}$ : cardiac index

aExpressed as a mean \pm standard deviation

Figure 1. Time to clinical deterioration (months), Subjects at risk, 3 CTD sub-groups $(n=25)$

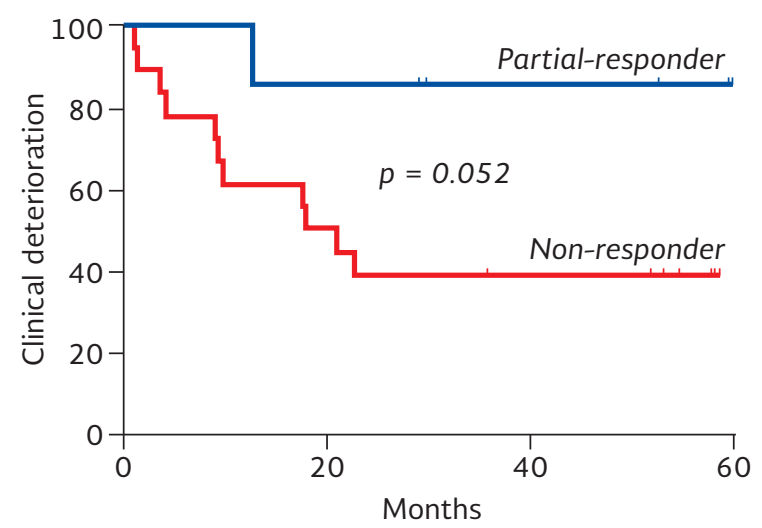

analyses between groups. We found that the SLE subgroup did yield a statistically significant difference in terms of clinical deterioration between partial responders and non-responders, using the Log-Rank test $(p=0.044)$. Figure 2 shows the survival analysis between groups.

Throughout follow-up, two deaths (8\%) were registered, one per group. The other clinical deterioration events were worsening of $\mathrm{PAH}$ in 5 patients (20\%); decrease in functional class in 18 (72\%); and a distance
Figure 2. Time to clinical deterioration (months), Subjects at risk, SLE sub-group $(n=16)$

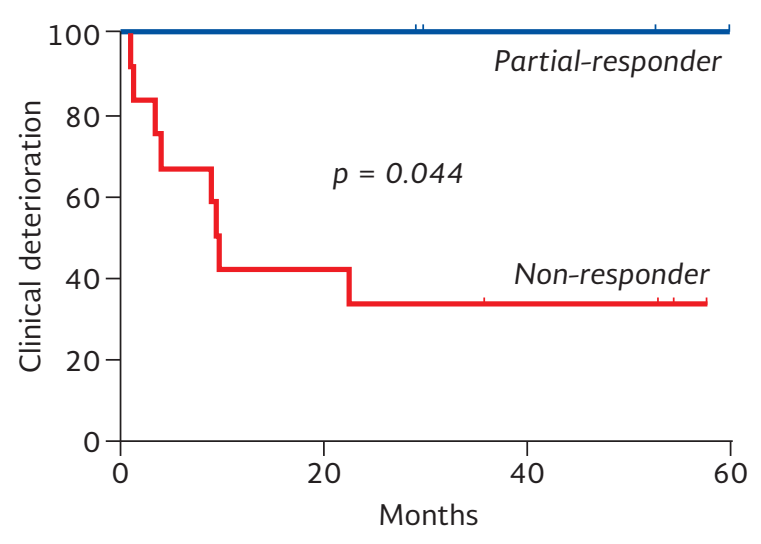

decrease in the 6MWT in 23 (92\%), with an average decrease on the number of meters walked, were $38 \%$.

\section{DISCUSSION}

This study showed that patients with PAH-CTD are partially responding to inhaled iloprost in the APVT, had a better prognosis in terms of remaining free of clinical deterioration for a longer time period than non-responders. 
The definition of response in the APVT used for this study differs from that used in recent PAH diagnostic and treatment guidelines ${ }^{1,2}$. None of the patients in our cohort had a classic response in the test, a finding similar to that reported by Halliday et al. ${ }^{7}$ in a prospective analysis of 155 patients with group $1 \mathrm{PAH}$. In their study, $33 \%$ belonged in the PAH-CTD group, and only $16 \%$ of patients in this sub-group had a classic positive response in the APVT with nitric oxide; however, only responders with idiopathic PAH had improved survival compared with non-responders. Unlike that study, we only evaluated clinical deterioration and not survival, in patients with some degree of pulmonary vascular response.

Although in earlier studies, the APVT was used to identify patients with better survival and those who were candidates to treatment with the less expensive albeit effective option, calcium channel blockers ${ }^{4,6}$, our study results suggest that the APVT can predict outcomes such as clinical deterioration.

Approximately, only $13 \%$ of patients with idiopathic $\mathrm{PAH}$ respond in the APVT ${ }^{5,7}$ and this group, at most $80 \%$ still respond to calcium antagonist drugs after 1 year of follow-up ${ }^{1,5}$. To date, there are no prospective studies on PAH-CTD that evaluate the prognostic value of the APVT response in terms of other outcomes (different from survival), such as clinical deterioration.

Our results reflect that a partial positive response, defined as a decrease in mPAP of $\geq 10 \mathrm{mmHg}$ but an absolute value of $>40 \mathrm{mmHg}$ and no change in the cardiac output, confers a better prognosis in terms of clinical deterioration in this sub-group of patients. These findings are similar to those reported by Malhotra et al. [8] in a retrospective cohort of 80 patients with group $1 \mathrm{PAH}$, in which $29 \%$ of cases had $\mathrm{PAH}-\mathrm{CTD}$, and an $\geq 12 \%$ decrease in mPAP with inhaled nitric oxide was an independent survival predictor when compared with non-responding patients, including those that were never treated with calcium channel blockers.

In other pulmonary hypertension groups, observing some degree of response in the APVT suggests a better prognosis. In a prospective study of 103 patients with chronic thromboembolic pulmonary hypertension (group IV), Skoro-Sajer et al. ${ }^{11}$ demonstrated that decreasing the mPAP to $>10.4 \%$ during the APVT with inhaled nitric oxide predicted longterm survival and longer lung transplant-free period in adult patients that underwent pulmonary endarterectomy.

A possible explanation to why patients with a partial pulmonary vascular response had less severe pulmonary hypertension and higher cardiac output may be the presence of protective antibodies (anti-U1-RNP). Although their role in disease has not been established in cohort studies, these antibodies predict better long-term survival in the three types of CTD ${ }^{12,13}$ and, perhaps, the pulmonary vascular response is an epiphenomenon of a phenotype associated to milder disease (less right ventricular remodeling).

Our study has several limitations such as the small patient number, a bias due to referral to a tertiary care hospital, and the convenience analysis of patients in a single center. However, despite these observations, there are no reports of prospective studies characterizing this specific sub-group of patients and results require validation by future studies.

Patients with a partial positive response in the acute pulmonary vasodilator test with inhaled iloprost had a better prognosis, remaining free of clinical deterioration during a longer time period, compared with non-responding patients.

\section{ACKNOWLEDGMENTS}

The authors gratefully acknowledge Deborah AlemánHoey, MD, for her assistance in the review of this manuscript. This study was funded by the Department of Cardiopulmonary Disease of the Instituto Nacional de Ciencias Médicas y Nutrición Salvador Zubirán, Mexico City, Mexico.

\section{REFERENCES}

1. Galiè N, Humbert M, Vachiery JL, et al. 2015 ESC/ERS guidelines for the diagnosis and treatment of pulmonary hypertension: the joint task force for the diagnosis and treatment of pulmonary hypertension of the European society of cardiology (ESC) and the European respiratory society (ERS): endorsed by: association for European paediatric and congenital cardiology (AEPC), international society for heart and lung transplantation (ISHLT). Eur Heart J. 2016;37:67-119.

2. Hoeper MM, Bogaard HJ, Condliffe R, et al. Definitions and diagnosis of pulmonary hypertension. J Am Coll Cardiol. 2013; 62:D42-50. 
3. Chung L, Liu J, Parsons L, et al Characterization of connective tissue disease-associated pulmonary arterial hypertension from REVEAL: identifying systemic sclerosis as a unique phenotype. Chest. 2010;138:1383-94.

4. Rich S, Kaufmann E, Levy PS. The effect of high doses of calcium-channel blockers on survival in primary pulmonary hypertension. N Engl J Med. 1992;327:76-81.

5. Sitbon $\mathrm{O}$, Humbert $\mathrm{M}$, Jaïs $\mathrm{X}$, et al. Long-term response to calcium channel blockers in idiopathic pulmonary arterial hypertension. Circulation. 2005;111:3105-11

6. Montani D, Savale L, Natali D, et al. Long-term response to calcium-channel blockers in non-idiopathic pulmonary arterial hypertension. Eur Heart J. 2010;31:1898-907.

7. Halliday SJ, Hemnes AR, Robbins IM, et al. Prognostic value of acute vasodilator response in pulmonary arterial hypertension: beyond the "classic" responders. J Heart Lung Transplant. 2015; 34:312-8.

8. Malhotra R, Hess D, Lewis GD, Bloch KD, Waxman $A B$, Semigran MJ. Vasoreactivity to inhaled nitric oxide with oxygen predicts long-term survival in pulmonary arterial hypertension. Pulm Circ. 2011;1:250-8

9. Jing ZC, Jiang $X$, Han ZY, et al. lloprost for pulmonary vasodilator testing in idiopathic pulmonary arterial hypertension. Eur Respir J. 2009;33:1354-60.

10. Badesch DB, Raskob GE, Elliot CG, et al. Pulmonary arterial hypertension. Baseline characteristics from the REVEAL Registry. Chest. 2010;137:376-87.

11. Skoro-Sajer N, Hack N, Sadushi-Koliçi R, et al. Pulmonary vascular reactivity and prognosis in patients with chronic thromboembolic pulmonary hypertension: a pilot study. Circulation. 2009; 119:298-305.

12. Sobansky V, Giovannelli J, Lynch BM, et al. Characteristics and survival of anti-U1 RNP antibody-positive patients with connective tissue disease-associated pulmonary arterial hypertension. Arthritis Rheumatol. 2016;68:484-93.

13. Huang C, Li M, Liu Y, Wang Q, Guo X, Zhao J. Baseline characteristics and risk factors of pulmonary arterial hypertension in systemic lupus erythematosus patients. Medicine. 2016;95:1-7. 